\title{
A NONLINEAR MODEL OF AGE AND SIZE-STRUCTURED POPULATIONS WITH APPLICATIONS TO CELL CYCLES
}

\author{
S. J. CHAPMAN ${ }^{1}$, M. J. PLANK ${ }^{92}$, A. JAMES ${ }^{2}$ and B. BASSE ${ }^{2}$
}

(Received 15 July, 2007; revised 20 October, 2007)

\begin{abstract}
The Sharpe-Lotka-McKendrick (or von Foerster) equations for an age-structured population, with a nonlinear term to represent overcrowding or competition for resources, are considered. The model is extended to include a growth term, allowing the population to be structured by size or weight rather than age, and a general solution is presented. Various examples are then considered, including the case of cell growth where cells divide at a given size.

2000 Mathematics subject classification: primary 37N25; secondary 92D25.

Keywords and phrases: age-structure, size-structure, populations, cell cycle, mathematical model.
\end{abstract}

\section{Introduction}

An in-depth understanding of the perturbations to the cell cycle of in vitro cancer cell populations by cancer therapies is crucial if one is to gain an understanding of the more complicated mechanisms of cancer treatments in patients. An age-structured population model of Basse and Ubezio [4] and Basse et al. [2] was used to keep track of the number density of in vitro cells within individual phases of the cell cycle both perturbed and unperturbed by a range of cancer therapies. Model results were compared to experimental outcomes by measuring the DNA distributions of the cell populations. It was assumed in the model that the DNA content of a particular cell depended on the 'age' of the cell where age is defined to be the time since arrival in that phase. No overcrowding was represented but this is a component that must not be overlooked in the in vivo situation.

\footnotetext{
'Mathematical Institute, Oxford University, Oxford, UK; email: chapman@maths.ox.ac.uk.

${ }^{2}$ Biomathematics Research Centre, University of Canterbury, Christchurch, New Zealand, email:

m.plank@math.canterbury.ac.nz, a.james@math.canterbury.ac.nz,b.basse@math.canterbury.ac.nz.

(C) Australian Mathematical Society 2007, Serial-fee code 1446-1811/07
} 
In this paper we aim to extend this work by investigating age and size-structured models with birth and death rates non-linearly dependent on total population number (that is, an overcrowding effect) and age or size. Here size is a generic term but in cancer cells the most applicable measure of size is DNA content. We initially derive a general age-structured model and extend this to a general size structured model. With a small modification this general size structured model can be applied to cell populations where the parent cell divides and is replaced by two daughter cells. We show the equivalence of these models under certain conditions.

In Sections 2.1 and 2.2 we consider the steady state and the general initial-value problem solutions respectively. In the latter case we are able to reduce an integropartial differential equation to a first-order ordinary differential equation. In Section 2.3 we consider particular examples for the dependence of the mortality rate on the total population which allow this equation to be solved explicitly. These include the case in which mortality is linearly dependent on total population size as considered in Gurtin and MacCamy [11]. In Section 2.4, we consider some specific examples of birth function, which allow us to get a feel for the behaviour of solutions. We start by considering the case of constant birth function (considered previously in Norhayati and Wake [16]) for both the age- and weight-structured case. We then consider the age-structured case in which members of a population give birth at a specific age, so that the birth rate function is a Dirac delta-function ( $\delta$-function), but in which the parent survives (and therefore continues to be a drain on resources). Finally, we consider a model for cell populations in which the population is structured by DNA content and cells divide when they reach a given DNA content, so that the birth rate function is again a $\delta$-function, but the parent cell is removed on division.

The study of age-structured population models has a long history. Amongst the first continuous models were those by Sharpe and Lotka [17], McKendrick [14] and von Foerster [19]. These authors assumed linear functions for the mortality rate of the population and a maternity or birth function through which the new population of age zero is produced. The resulting model has been extensively studied and details can be found in, for example, Hoppensteadt [13] or Murray [15]. Linear models of this type lead to a population which, in general, either grows exponentially or dies out.

More general models where the mortality rate at a given age may have some dependence on the total population size have also been studied. In particular, Gurtin and MacCamy [11] extended the linear model to include a nonlinear term in which the mortality rate is proportional to the overall size of the population. This term can be attributed to the effects of overcrowding or competition for resources and has the effect of limiting the otherwise exponentially growing solution to a finite steady state in many cases. A good overview of these models is given by Webb [20]. 
1.1. A general age-structured model If we denote by $u(a, t)$ the number density of individuals of age $a$ at time $t$, then a general age-structured model for the evolution of $u$ may be written as (Cushing [9], Gurtin and MacCamy [11])

$$
\begin{aligned}
u_{1}+u_{a} & =-\mu(a, U) u, \\
u(0, t) & =\int_{0}^{\infty} b(a, U) u(a, t) d a, \\
u(a, 0) & =u_{0}(a), \\
\text { where } U & =\int_{0}^{\infty} \xi(a) u(a, t) d a,
\end{aligned}
$$

$u_{0}(a)$ is the number density of individuals of age $a$ initially, $\mu(a, U)$ is the mortality rate, and $b(a, U)$ is the birth or renewal function, representing the rate of production of individuals of age zero from individuals of age $a$. If $\xi(a) \equiv 1$, then $U$ is the total size of the population. In general, $\xi(a)$ is a weight function that represents different nutrient uptake rates by individuals of different ages. For example, taking $\xi(a)$ to be an increasing function of $a$ would reflect the case where older individuals consume more resources, and hence have a greater impact on the rest of the population, via the mortality rate $\mu(a, U)$. For simplicity, $U$ is referred to throughout as the effective total population size. In this general case, both the mortality rate and the birth rate may be functions of age and the total population size but they are linear in $u$, the population density.

1.2. A general size-structured model Many populations are not structured specifically by age but more generally by size or weight. In these cases, the governing equations must be extended to include a growth term. This extension has been used previously by Botsford $e$ t al. [6] to model sea-urchin populations, by Takada and Caswell [18] to study optimal maturity size and by Coombes $e t$ al. [8] to study the density distributions of trees. However, no term was used to represent overcrowding in these studies.

Suppose the rate of gain of weight $w$ of an individual is $r(w)$, so that for each individual

$$
\frac{\mathrm{d} w}{\mathrm{~d} t}=r(w) .
$$

Common models for weight as a function of time are exponential growth (for example, as a simple model for cell size) or von Bartalanffy growth, in which the growth rate slows as the individual ages and there is a maximum weight that an individual can reach (which has been used, to model the size of an individual creature (Hilborn and Walters [12])). Both of these can be modelled by choosing, for example,

$$
r(w)=1+\alpha w,
$$


in which $\alpha>0$ gives exponential growth and $\alpha<0$ gives von Bartalanffy-type growth. The general weight-structured population model is given by

$$
\begin{aligned}
u_{t}+(r(w) u)_{w} & =-\mu(U, w) u, \\
U & =\int_{0}^{\infty} \xi(w) u(w, t) d w, \\
u(0, t) r(0) & =\int_{0}^{\infty} b(w, U) r(w) u(w, t) d w, \\
u(w, 0) & =u_{0}(w) .
\end{aligned}
$$

In writing down the renewal condition (1.7), we have used the convention that $w$ is not the actual weight but the weight gained after birth, which allows the renewal condition to be imposed at $w=0$. We suppose that $r(0)>0$ and $r(w) \geq 0$ for all $w$, so that an individual's weight will never decrease, and also that $\lim _{w \rightarrow \infty} r(w) / w$ is finite, so that an individual cannot grow to an infinite weight in a finite time.

\subsection{Equivalence of the general age-structured and size-structured models Al-} though it appears at first sight that the weight-structured model (1.5)-(1.8) is more general than the age-structured model (1.1)-(1.4), in fact they are equivalent, as we now show. We first define $v=r(w) u$ as the population per unit age (recall that $u$ is the population per unit weight), and multiply Equation (1.5) by $r(w)$ to obtain

$$
v_{t}+r(w) v_{w}=-\mu(U, w) v .
$$

The characteristics are given by

$$
\frac{d w}{d t}=r(w), \quad \text { or } \quad t-R(w)=\text { constant }
$$

where $R(w)=\int_{0}^{w} r(x)^{-1} d x$ is the time taken for an individual to grow from weight zero to weight $w$, that is, the age of an individual of weight $w$. Note that $R(0)=0$ and $R$ is a strictly increasing function. The characteristics are illustrated in Figure 1.

If the growth rate is strictly positive (that is, $r(w)>0$ for all $w$ ), then $R(w) \rightarrow \infty$ as $w \rightarrow \infty$. However, if $r\left(w_{m}\right)=0$ for some $w_{m}$, then there is a limiting weight $w_{m}$ to which individuals can grow. In this case, we require the initial condition to satisfy $u_{0}(w)=0$ for $w>w_{m}$, in which case $u(w, t)$ is zero for $w>w_{m}$. In both cases, the inverse $R^{-1}$ exists and is strictly increasing on the positive real line: $R^{-1}(t)$ is the weight of an individual of age $t$.

We now write $v$ as a function of age $a=R(w)$ rather than $w$, and write $\hat{\mu}(U, a)=$ $\mu(U, w)$ to find

$$
v_{t}+v_{a}=-\hat{\mu}(U, a) v
$$




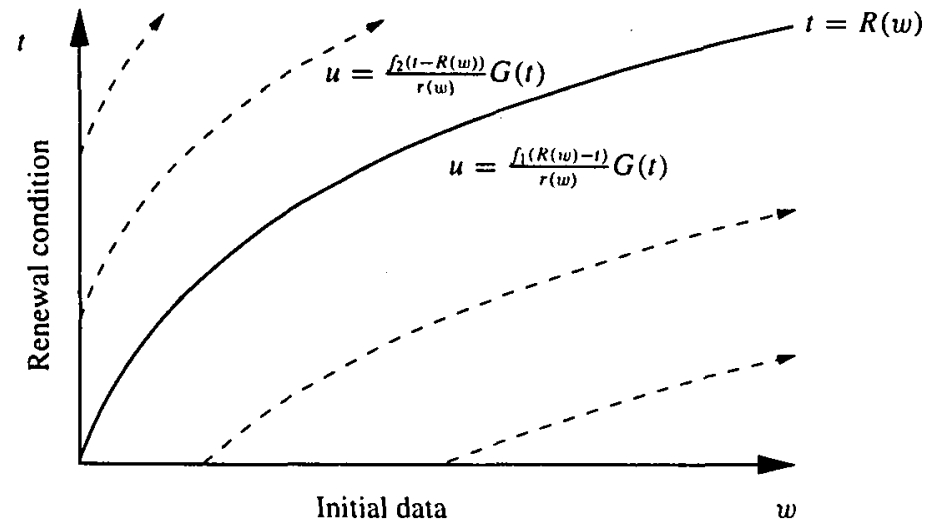

FIGURE 1. The characteristics for the weight-structured model. The solution propagates along characteristics, which are the curves $t-R(w)=$ constant, as determined by the growth rate $r(w)$. The solution space is divided into two regions: for $t<R(w)$, the solution is determined by the initial density of individuals of weight $R(w)-t$; for $t>R(w)$, the solution is determined by the birth rate at time $t-R(w)$. The function $G(t)$ represents the effects of the death rate, causing the solution to decay with time.

The effective total population size is given by

$$
U=\int_{0}^{\infty} \xi(w) u d w=\int_{0}^{\infty} \xi\left(R^{-1}(a)\right) r(w) u d a=\int_{0}^{\infty} \hat{\xi}(a) v d a,
$$

while the renewal condition is

$$
v(0, t)=\int_{0}^{\infty} b(w, U) v d w=\int_{0}^{\infty} r(w) b(w, U) v d a=\int_{0}^{\infty} \hat{b}(a, U) v d a,
$$

where

$$
\hat{b}(a, U)=b\left(R^{-1}(a), U\right) r\left(R^{-1}(a)\right)
$$

is the birth rate of individuals of age $a$. Finally, the initial condition is

$$
v(a, 0)=r(w) u(w, 0)=r\left(R^{-1}(a)\right) u_{0}\left(R^{-1}(a)\right) .
$$

1.4. A general model for cell populations With the extension of the age-structured population model to a size-structured population model, it is now possible to use this system to model populations of independently growing cells that are competing for limited resources. A particularly important example of this is a population of cancer cells, which possess some genetic defect freeing them from the normal restraints on proliferation and growth. The cells are thus effectively competing with one another for nutrients, and a limited availability of nutrients generally imposes a maximum diameter on a tumour that would otherwise continue to grow exponentially Folkman [10]. 
Cells reproduce by duplicating their genetic material and then splitting into two daughter cells; this process is part of the cell cycle, common to all cells. The cell cycle comprises four main phases. In the $S$-phase, the cell's DNA is replicated, whilst the physical division of the nucleus and then the cell itself occur during the $M$-phase, or mitosis phase. Between these two phases are two gap phases, $G_{1}$ and $G_{2}$ (Alberts et al. [1]). A system of the form (1.1)-(1.4) has been used previously to model cell growth by Chen $e t$ al. [7]. The aim of the study was to examine the effects of ionizing radiation damage through a specific mortality term: the population was age-structured and no effects due to competition were included. Basse $e t$ al. [3] studied a fourcompartment model (one compartment for each phase of the cell cycle) of cancer cell growth in which the population was structured by DNA content. During the $S$-phase, DNA content was assumed to increase at a constant rate, with no change in DNA content during the other phases, until mitosis occurs (in the $M$-phase) at which point the cell divides, producing two daughter cells, each with half the DNA content of the parent. In a further paper, Basse et al. [5] considered a single compartment model of cell growth in plankton, which allowed for a non-uniform growth rate. Again, no overcrowding effects were considered in these studies.

A modification to the basic model (1.5)-(1.8) for a size-structured population is necessary to take account of the fact that a cell does not give birth to a daughter cell, but in fact divides to form two daughter cells, both of 'size' $w=0$ (recall that $w$ is the DNA content gained after birth, not total DNA content). Each time a parent cell divides the parent cell effectively ceases to exist, leading to an additional loss term in the equation for $u(w, t)$ (see, for example, Chen et al. [7]). Since this extra term is linear in $u$, it can be incorporated into the mortality rate $\mu(U, w)$. Thus in Equation (1.5), the total loss rate is

$$
\mu(U, w)=\mu_{\text {death }}(U, w)+\frac{b(w, U) r(w)}{B},
$$

where $B$ daughter cells are produced for every parent (usually $B=2$ ).

\section{Age-structured model with mortality rate $\mu(U, a)=q(U)+p(a)$ and birth rate $b=b(a)$}

Although there is much literature discussing the existence and uniqueness of solutions of the general model (1.1)-(1.4), there is little work that looks at the form of the solutions for particular cases of either the mortality and birth functions. Here we study (1.1)-(1.4) for the particular case in which the mortality function separates into the sum of a function of total population $U$ and a function of age $a$. We allow the birth rate to be an arbitrary function of age, but choose it to be independent of the total population size, so there is no effect on the birth rate due to overcrowding. 
These are simplifying assumptions that allow an analytical solution to the problem. Nevertheless, these choices of $\mu$ and $b$ are sufficiently general to model overcrowding and competition for resources, and to include the additional loss term in (1.10) due to parent cells ceasing to exist. That is, if the mortality function of the age-structured population is separated into the sum of a function of total population $U$ and a function of age $a$ then the cell population model equations are equivalent to the age-structured population. We therefore proceed with analysis of the age-structured population alone.

Specifically we let $\mu(U, a)=q(U)+p(a)$, and the birth rate $b=b(a)$. As stated in the introduction, if there is an additional loss term due to parent cells ceasing to exist on division as in (1.10), then we incorporate this into $p(a)$. Thus our equations are

$$
\begin{aligned}
u_{t}+u_{a} & =-(q(U)+p(a)) u=-(g(t)+p(a)) u, \\
U & =\int_{0}^{\infty} \xi(a) u(a, t) d a, \\
u(0, t) & =\int_{0}^{\infty} b(a) u(a, t) d a, \\
u(a, 0) & =u_{0}(a),
\end{aligned}
$$

where, since $U$ is a function of $t$ only, we have written $q(U)=g(t)$. In Section 2.1 we briefly consider the steady state. Then, in Section 2.2 we consider the general initialvalue problem, and reduce (2.1)-(2.4) to a first-order ordinary differential equation. The technique is based on solving (2.1)-(2.4) assuming that $g$ is known, and then applying a consistency condition to determine $g$.

2.1. Steady state Before we examine the general initial-value problem, we first briefly examine the steady-state solutions. In steady state, $g$ is a constant and

$$
u=A \exp \left(-g a-\int_{0}^{a} p(z) d z\right)=A P(a) e^{-g a},
$$

where

$$
P(a)=\exp \left(-\int_{0}^{a} p(z) d z\right)
$$

Then

$$
1=\int_{0}^{\infty} b(a) P(a) e^{-g a} d a=\overline{b P}(g),
$$

where an overbar denotes the Laplace transform. Equation (2.6) determines $g$, and then $A$ can be determined by solving

$$
g=q(U)=q\left(A \int_{0}^{\infty} \xi(a) P(a) e^{-g a} d a\right) .
$$

Note that if $b \geq 0$ then the solution for $g$ is unique, since $\overrightarrow{b P}^{\prime}(g) \leq 0$, so that $\overline{b P}(g)$ is monotonic. If $q$ is monotonic in $U$ then the solution for $A$ will be unique. 
2.2. Solution to the initial value problem We now solve the general initial-value problem. Making the change of variables

$$
w(a, t)=u(a, t) \exp \left(\int_{0}^{t} g(z) d z\right),
$$

it may be seen from (2.1) that $w$ satisfies

$$
w_{t}+w_{a}=-p(a) w(a, t),
$$

with boundary and initial conditions

$$
w(0, t)=\int_{0}^{\infty} b(a) w(a, t) d a, \quad w(a, 0)=u_{0}(a) .
$$

The total effective population size is

$$
U=\int_{0}^{\infty} \xi(a) u(a, t) d a=\exp \left(-\int_{0}^{t} g(z) d z\right) h(t),
$$

and the unknown function $g(t)$ satisfies the integral equation

$$
g(t)=q(U)=q\left(\exp \left(-\int_{0}^{t} g(z) d z\right) h(t)\right),
$$

where $h(t)=\int_{0}^{\infty} \xi(a) w(a, t) d a$.

Note that $w$ satisfies a linear equation. By the theory of linear age-structured populations, it is expected that, provided the birth and mortality functions are sufficiently smooth, the total effective population size $h(t)$ in the $w$-variable will converge asymptotically to exponential growth or decay $C e^{\lambda t}$ for large $t$ (Webb [20], Chapter 1). Periodic solutions are only possible with a non-smooth birth/mortality function, such as the delta birth function considered in Section 2.4.

The characteristics of (2.1) are simply $t-a=$ constant, as illustrated in Figure 2, and we may solve for $w$ to give

$$
w(a, t)= \begin{cases}f_{1}(a-t) P(a) & \text { if } t<a, \\ f_{2}(t-a) P(a) & \text { if } t>a,\end{cases}
$$

where $P(a)$ is given by (2.5). The initial condition determines $f_{1}$ as

$$
f_{1}(a)=\frac{u_{0}(a)}{P(a)} .
$$

The boundary condition at $a=0$ gives an integral equation for $f_{2}$,

$$
f_{2}(t)-\int_{0}^{t} b(a) f_{2}(t-a) P(a) d a=\int_{t}^{\infty} \frac{b(a) u_{0}(a-t) P(a)}{P(a-t)} d a=F(t),
$$

say, which can be solved by taking a Laplace transform to give

$$
\bar{f}_{2}=\frac{\bar{F}}{(1-\overline{b P})} \text {. }
$$




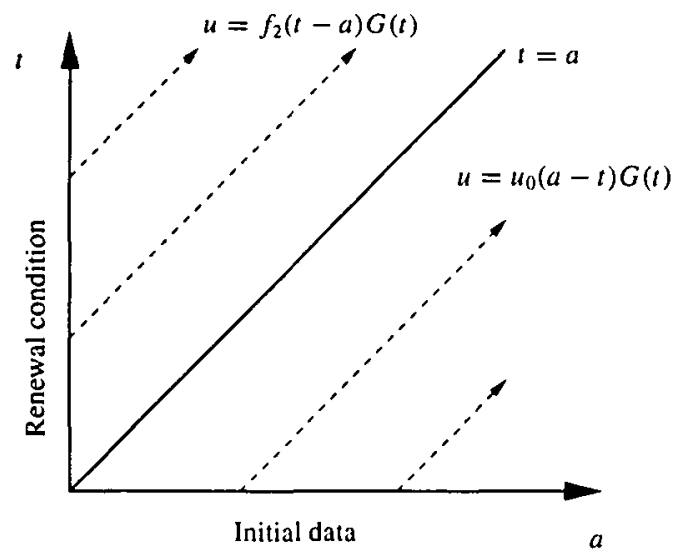

FIGURE 2. Diagram of the characteristics for the age-structured model. The solution propagates along characteristics, which are the straight lines $t-a=$ constant. The solution space is divided into two regions: for $t<a$, the solution is determined by the initial density of individuals of age $a-t$; for $t>a$, the solution is determined, via the renewal condition, by the birth rate at time $t-a$. The function $G(t)$ represents the effects of the death rate, causing the solution to decay with time.

Thus

$$
f_{2}(t)=\frac{1}{2 \pi i} \int_{c-i \infty}^{c+i \infty} \frac{\bar{F}(s)}{1-\overline{b P}(s)} e^{s t} d s .
$$

Now, from Section 2.1, we know that the equation $\overline{b P}(s)=1$ has exactly one real root, at $s=s^{*}$ say, which is the steady-state value of $g$. The residue from this pole gives the steady-state solution for $u(a, t)$. There will also be complex roots of $\overline{b P}(s)=1$, as well as possible poles of $\bar{F}(s)$. In addition there could be a continuous spectrum due to a branch point etc. If the real part of all the other poles is less than $s^{*}$, then the solution will converge to the steady state for large time. This is the case when $b P$ is constant, which we consider in Examples 1 and 2. If another complex conjugate pair of poles dominates, then the solution may oscillate. In the case where $b$ is a $\delta$-function, which we consider in Examples 3 and 4, all the roots of $\overline{b P}(s)=1$ have the same real part, which enables them all to play a role in the large-time behaviour, giving oscillating solutions.

Now that $f_{2}$ has been found, we can determine the function $h(t)$ as:

$$
h(t)=\int_{0}^{\infty} \frac{\xi(a) u_{0}(a) P(t+a)}{P(a)} d a+\int_{0}^{t} \xi(t-x) f_{2}(x) P(t-x) d x .
$$

Writing $g=f^{\prime} / f, h(t)=\int_{0}^{\infty} \xi(a) w(a, t) d a$ and choosing $f(0)=1$ without loss of generality, (2.8) becomes

$$
f^{\prime}=f q\left(\frac{h(t)}{f}\right)
$$


Thus we have reduced the original integro-partial differential equation (2.1) to the first-order ordinary differential equation (2.13). For certain choices of $q(U)$, Equation (2.13) can be solved explicitly; we consider some of these in Section 2.3.

To summarise, define $G(t)=\exp \left(-\int_{0}^{t} g(z) d z\right)=1 / f(t)$. Then the solution is

$$
u(a, t)= \begin{cases}u_{0}(a-t) G(t) \frac{P(a)}{P(a-t)} & \text { if } t<a, \\ f_{2}(t-a) G(t) P(a) & \text { if } t>a,\end{cases}
$$

where $P$ is given by (2.5), $f_{2}$ by (2.11) and $G$ is obtained by solving the ODE (2.13).

Before we go on to consider some specific examples of the dependence of the mortality rate on the total population in which Equation (2.13) can be integrated explicitly, we first note that, in the case in which mortality is independent of age, so that $p(a) \equiv 0$ and $\mu(U, a)=q(U)$, we have $P(a) \equiv 1$. In this case, denoting the initial effective size of the population by $U_{0}$, the first term in Equation (2.12) for $h(t)$ is constant:

$$
h(t)=U_{0}+\int_{0}^{t} \xi(t-x) f_{2}(x) d x .
$$

2.3. Examples investigating different mortality rates Henceforth assume that $\xi(a) \equiv 1$, so that all individuals have the same impact on overcrowding regardless of size.

If mortality is linear in effective population size $U$, that is,

$$
\mu(U, a)=\mu_{1}+\mu_{2} U(t)+p(a),
$$

then Equation (2.13) for $f$ is a linear equation which can be integrated, with initial condition $f(0)=1$, to give

$$
g(t)=\frac{f^{\prime}}{f}=\mu_{1}+\frac{\mu_{2} h(t)}{e^{\mu_{1} t}\left(1+\mu_{2} \int_{0}^{t} e^{-\mu_{1} y} h(y) d y\right)} .
$$

If the mortality has a power-law dependence on population size, that is,

$$
\mu(U, a)=\alpha U^{n}+p(a),
$$

then Equation (2.13) is separable and can again be solved explicitly to give that

$$
g(t)=q\left(\frac{h(t)}{f}\right)=\frac{\alpha h(t)^{n}}{\left(1+\alpha n \int_{0}^{t} h(z)^{n} d z\right)} .
$$

If mortality is inversely dependent on population size, that is, $\mu(U, a)=\mu_{1}+$ $\mu_{2} / U+p(a)$, then Equation (2.13) is linear in $G=1 / f$ and can be integrated to give

$$
g(t)=q(h(t) G(t))=\mu_{1}+\frac{\mu_{2} e^{\mu_{1} t}}{h(t)\left(1-\mu_{2} \int_{0}^{t} \frac{e^{\mu_{1} y}}{h(y)} d y\right)} .
$$


2.4. Examples investigating different birth and growth functions We now consider some examples of particular birth functions to build up some intuition about the solution developed in Section 2.2. Throughout we choose a mortality rate that is linear in $U\left(q(U)=\mu_{1}+\mu_{2} U\right)$. For Examples 1-3, we choose $p(a)=0$ so that $P(a) \equiv 1$ and $h(t)$ is given by (2.15). When we consider a model for cell populations in Example 4, the mortality rate depends on $a$ through the fact that the parent is removed on division.

EXAMPLE 1 (Constant growth rate; constant birth rate). We consider first the case in which birth rate is constant (previously considered in [16]), so that $b(a) \equiv B$. Then the solution (2.14) for $u$ is

$$
u(a, t)= \begin{cases}\frac{\left(B-\mu_{1}\right) u_{0}(a-t) e^{-\mu_{1} t}}{\mu_{2} U_{0} e^{\left(B-\mu_{1}\right) t}+B-\mu_{1}-\mu_{2} U_{0}} & \text { if } t<a, \\ \frac{\left(B-\mu_{1}\right) B U_{0} e^{\left(B-\mu_{1}\right) t-B a}}{\mu_{2} U_{0} e^{\left(B-\mu_{1}\right) t}+B-\mu_{1}-\mu_{2} U_{0}} & \text { if } t>a .\end{cases}
$$

If $B<\mu_{1}$ (that is, the birth rate is less than the basic mortality rate) then $u(a, t) \rightarrow 0$ as $t \rightarrow \infty$ and the population will eventually die out. If $B>\mu_{1}$ then as $t \rightarrow \infty$ the solution will, for any initial condition, tend to the positive steady state

$$
u_{\infty}(a)=\frac{B\left(B-\mu_{1}\right) e^{-B a}}{\mu_{2}} .
$$

From (2.7), the total population is given by

$$
U(t)=\frac{U_{0}\left(B-\mu_{1}\right) e^{\left(B-\mu_{1}\right) t}}{\mu_{2} U_{0} e^{\left(B-\mu_{1}\right) t}+B-\mu_{1}-\mu_{2} U_{0}},
$$

which converges monotonically to the steady state,

$$
U_{\infty}= \begin{cases}\frac{B-\mu_{1}}{\mu_{2}} & \text { if } B>\mu_{1}, \\ 0 & \text { if } B<\mu_{1} .\end{cases}
$$

More details of this special case can be found in [16]. Figure 3 shows the solution at various time intervals for a "top hat" initial condition, $u_{0}(a)=H(a)-H(a-1)$, where $H$ is the Heaviside step function. As expected for this $B>\mu_{1}$ case, the initial condition propagates along the characteristics $t-a=$ constant, keeping its original shape, and the solution converges to the steady state, which is an exponentially decreasing function of age. 


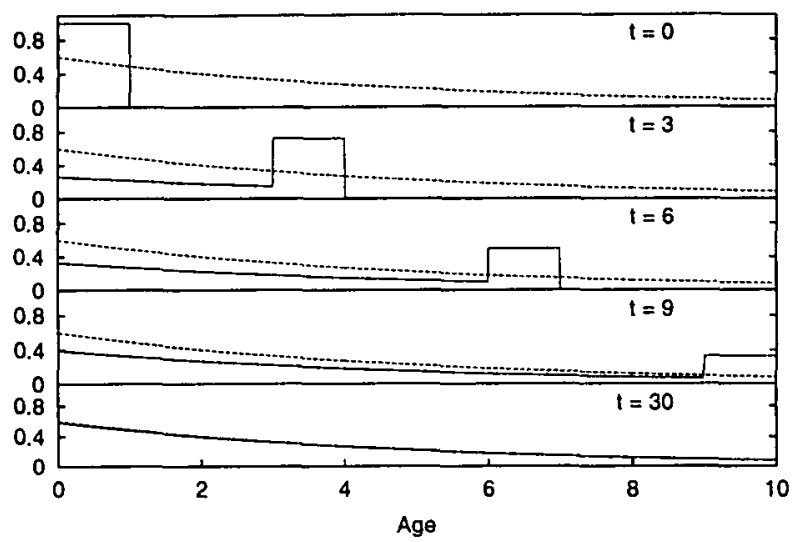

FIGURE 3. Population density against age at successive values of time for an age-structured population with a constant birth function, $b(a) \equiv B=0.2$. The mortality rates are $\mu_{1}=\mu_{2}=0.05$. The "top hat" initial condition converges to the steady state (dashed line).

Finally, we note that when $b(a) \equiv B$ and $p(a) \equiv 0$, an equation for the total population can be written down for general $q(U)$ by integrating the original equation with respect to $a$ :

$$
U^{\prime}+(q(U)-B) U=0 .
$$

EXAMPLE 2 (Non-constant growth rate; constant birth rate). We examine the effect of non-uniform growth on the example of Section 1. We choose $r$ to be given by

$$
r(w)=1+\alpha w,
$$

where $\alpha>0$ gives exponential growth and $\alpha<0$ gives von Bartalanffy-type growth. In the special case, $\alpha=0$, the growth rate $r(w)$ is a constant, $R(w)=w$ and the solution collapses to the age-structured case. In the general case, the solution (2.14) is given by

$$
u(w, t)= \begin{cases}\frac{f_{1}(R(w)-t)}{1+\alpha w} G(t) & \text { if } t<R(w), \\ \frac{f_{2}(t-R(w))}{1+\alpha w} G(t) & \text { if } t>R(w),\end{cases}
$$

where

$$
\begin{aligned}
& G(t)=\left[e^{\mu_{1 t}}+\frac{\mu_{2}}{\alpha+B}\left[\frac{U_{0} \alpha-V_{0} B}{\mu_{1}}\left(e^{\mu_{1} t}-1\right)+\frac{B\left(U_{0}+V_{0}\right)}{\alpha+B-\mu_{1}}\left(e^{(\alpha+B) t}-e^{\mu_{1} t}\right)\right]\right]^{-1}, \\
& f_{1}(t)=e^{\alpha t} u_{0}\left(\frac{e^{\alpha t}-1}{\alpha}\right), \quad f_{2}(t)=B\left(U_{0}+V_{0}\right) e^{(\alpha+B) t}, \quad V_{0}=\alpha \int_{0}^{\infty} y u_{0}(y) d y
\end{aligned}
$$


If $\alpha<0$, there is a limiting weight, $w_{m}=-1 / \alpha$, and $u(w, t)$ is defined to be zero for $w>w_{m}$. In both the $\alpha>0$ and $\alpha<0$ cases, as $t \rightarrow \infty$, the solution tends to a steady state given by

$$
u_{\infty}(w)= \begin{cases}\frac{(\alpha+B)\left(\alpha+B-\mu_{1}\right)}{\mu_{2}(1+\alpha w)^{(2 \alpha+B) / \alpha}} & \text { if } B>0 \text { and } \alpha+B>\mu_{1}, \\ 0 & \text { otherwise. }\end{cases}
$$

Hence, provided the birth rate is strictly positive, the population will evolve to a positive steady state (with total population size $\left.U_{\infty}=\left(\alpha+B-\mu_{1}\right) / \mu_{2}\right)$ if $\alpha+B>\mu_{1}$, that is, if the sum of the birth rate and the slope of the linear growth rate is greater than the death rate. Otherwise the population will eventually die out.

Figure 4 shows the solutions at various time intervals for the $\alpha+B>\mu_{1}$ case with the same "top hat" initial condition as used in Example 1. Figure 4a is the case of exponential growth ( $\alpha>0)$; Figure $4 \mathrm{~b}$ is the case of von Bartalanffy growth $(\alpha<0)$. In both cases, the population evolves towards a positive steady state: if $\alpha>0$ then $u>0$ for all $w>0$; but if $\alpha<0$ then there is a maximum weight of $w_{m}=-1 / \alpha$ and $u=0$ for $w>w_{m}$. The behaviour is, at first glance, similar to that of the age-structured population (Figure 3), in which individuals always 'grow' at a constant rate. However, in the age-structured population, the travelling wave solutions retain the same shape as they propagate forward, whereas in the weight-structured population, the non-uniform growth rate can change the shape of the solution. In Figure $4 \mathrm{a}$ $(\alpha>0)$, the expcnential growth rate has the effect of stretching out the initial "top hat" as it grows (because individuals at the upper end are growing more rapidly than those at the lower end). In Figure $4 \mathrm{~b}(\alpha<0)$, the von Bartalanffy growth rate has the opposite effect, compressing the "top hat" as it moves towards the limiting weight.

EXAMPLE 3 (Constant growth rate; $\delta$-function birth rate with parent surviving). A common modelling assumption with regard to birth or renewal is that individuals give birth on reaching a certain age $a_{0}$, corresponding to a birth function $b$ of the form

$$
b(a)=B \delta\left(a-a_{0}\right),
$$

where $B$ is the number of offspring. Here we consider the case where the parent survives and continues to be a drain on resources, contributing to the total population $U$. In Example 4, we consider the case in which the parent is removed after reproducing, which is appropriate for models of cell division.

In the case where $b$ is a $\delta$-function, rather than solve Equation (2.10) using a Laplace transform, we may write directly

$$
f_{2}(t)=B^{n+1} u_{0}\left(a_{0}-\tau\right),
$$




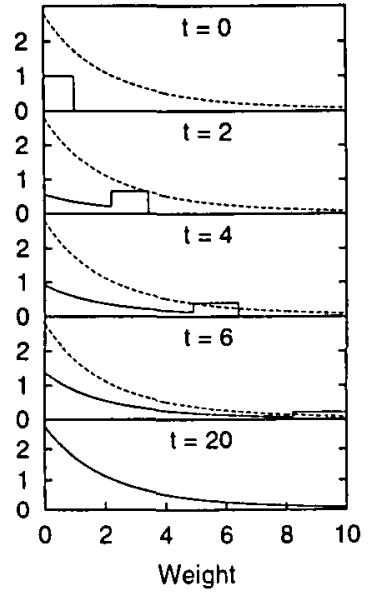

(a)

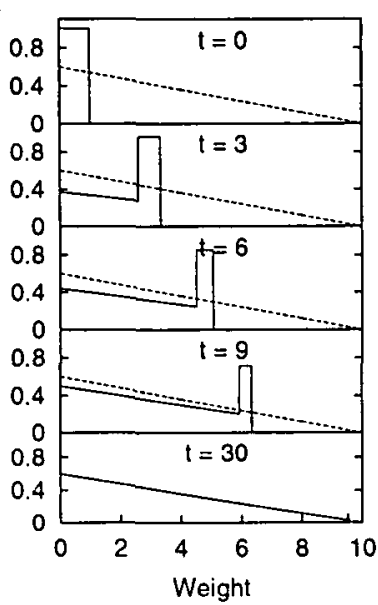

(b)

FIGURE 4. Population density against weight at successive values of time for a weight-structured population with a constant birth function, $b(a) \equiv B=0.3$. The mortality rates are $\mu_{1}=\mu_{2}=0.05$. The growth rate is $r(w)=1+\alpha w$ : (a) $\alpha=0.1$ (exponential growth); (b) $\alpha=-0.1$ (von Bartalanffy-type growth). For both cases the "top hat" initial condition converges to the steady state (dashed line).

where $n(t)$ and $\tau(t)$ are defined by by $t=n a_{0}+\tau$ with $0<\tau<a_{0}$, so that $n(t)$ is the number of birth cycles that have been completed at time $t$ and $\tau(t)$ is the time that has been spent in the current cycle. Then the solution for $G$ in Equation (2.14) is given by

$$
\begin{aligned}
\frac{1}{G}= & e^{\mu_{1} t}+\frac{\mu_{2}}{\mu_{1}}\left[U_{0}\left(e^{\mu_{1} t}-1\right)-\frac{B\left(1-B^{n}\right)}{1-B} \hat{U}_{0}-B^{n+1} \int_{0}^{\tau} u_{0}\left(a_{0}-x\right) d x\right. \\
& \left.+\frac{\hat{U}_{0}^{*} B e^{\mu_{1}\left(1-a_{11}\right)}\left(1-B^{n} e^{\left.-n \mu_{1} a_{t}\right)}\right)}{\left(1-B e^{-\mu_{1} a_{11}}\right)}+B^{n+1} e^{\mu_{1} \tau} \int_{0}^{\tau} e^{-\mu_{1} x} u_{0}\left(a_{0}-x\right) d x\right],
\end{aligned}
$$

where

$$
\hat{U}_{0}=\int_{0}^{a_{0}} u_{0}(x) d x, \quad \hat{U}_{0}^{*}=\int_{0}^{a_{11}} e^{\mu_{1} \cdot x} u_{0}(x) d x .
$$

Figure 5 shows the solutions for a "top hat" initial condition. The effect of the delta birth function is to create a new cohort of individuals each time the previous cohort reaches the reproduction age (that is, at $t=n a_{0}, n=1,2,3, \ldots$ ).

EXAMPLE 4 (Non-constant growth rate; $\delta$-function birth rate with parent removed). Our final example concerns the case of cell division, in which the parent is removed 


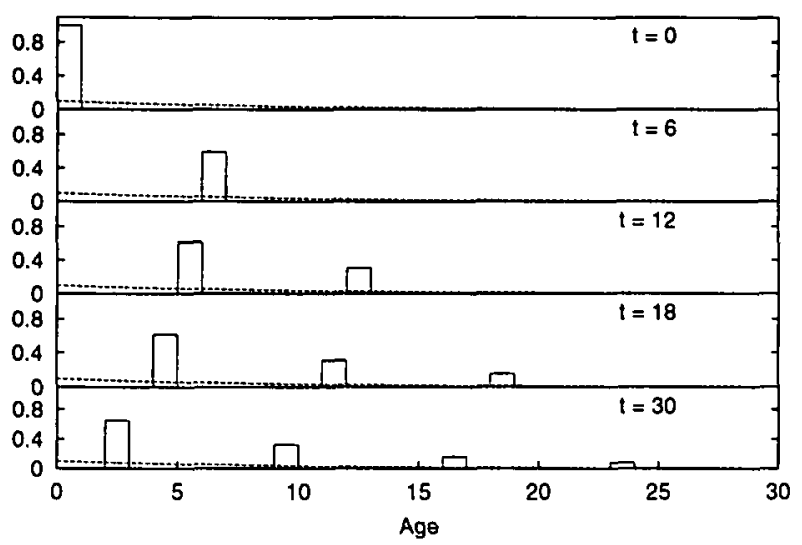

FIGURE 5. Population density against age at successive values of time with a delta birth function, $b(w)=B \delta\left(a-a_{0}\right)=2 \delta(w-7)$. The mortality rates are $\mu_{1}=\mu_{2}=0.05$. The "top hat" initial condition does not converge to the steady state (dashed line).

after dividing into two daughter cells. We choose the same growth function (2.17) as in Example 2. As in Example 3, we consider the case in which cells divide at a given size, so that $b(w)=B \delta\left(w-w_{0}\right)$ (of course, usually we are interested in the case $B=2$, but we leave $B$ arbitrary). The effect of the $\delta$-function on the mortality rate is to set $u=0$ for $w>w_{0}$ : no individuals can be older than the reproduction age $a_{0}=R\left(w_{0}\right)$. Thus

$$
P(a)= \begin{cases}1 & \text { if } a<a_{0} \\ 0 & \text { if } a>a_{0}\end{cases}
$$

Again, since $\hat{b}$ is a $\delta$-function, rather than solve Equation (2.10) using a Laplace transform, we may write directly

$$
f_{2}(t)=B^{n+1} f_{1}\left(a_{0}-\tau\right) .
$$

The solution for $G$ in Equation (2.14) is

$$
G(t)=\frac{e^{-\mu_{1} t}}{1+\mu_{2} \int_{0}^{t} e^{-\mu_{1} y} h(y) d y} .
$$

Figure 6 illustrates the propagation of the solution along the characteristic curves. When a characteristic reaches $w=w_{0}$ the delta birth function causes it to be multiplied by $B$ and returned to $w=0$ : this event corresponds to the division of a cell into $B$ daughter cells. 


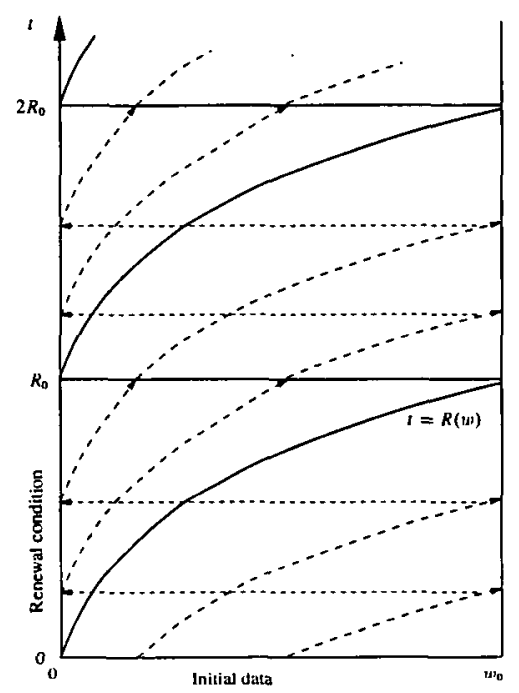

FIGURE 6. Diagram of the characteristics for the cell growth model. The solution propagates along characteristics, which are the curves $t-R(w)=$ constant. When the characteristics reach $w=w_{0}$, the delta renewal function is applied and the solution jumps back to $w=0$ (and is multiplied by $B$ ).

The nonzero steady state is

$$
u_{\infty}(w)=\frac{B \ln B\left(\ln B-\mu_{1} a_{0}\right)}{\mu_{2} a_{0}^{2}(B-1)} \frac{1}{r(w) B^{R(w) / a_{0}}},
$$

which is positive provided $\mu_{1} a_{0}<\ln B$. For any initial data of the form $u_{0}(w)=$ $K u_{\infty}(w)$ where $K>0$ is a constant, the solution retains the same shape for all time: $u(w, t)=K(t) u_{\infty}(w)$. Furthermore, $K(t) \rightarrow 1$ as $t \rightarrow \infty$, so the solution will converge to the steady state for initial data of this form.

For any other type of initial condition, the solution will instead converge to a periodic solution of period $a_{0}$. In particular, it can be shown that the solution at $t=n a_{0}$ where $n=0,1,2, \ldots$ (that is, at multiples of the cell cycle duration $a_{0}$ ) is given by:

$$
\begin{gathered}
u\left(w, n a_{0}\right)=\frac{(\sigma-1) B \mu_{1} u_{0}(w)}{B \mu_{1}(\sigma-1) \sigma^{-n}+\mu_{2} U_{0}^{*}\left(\sigma-\sigma^{1-n}\right)}, \text { where } \\
U_{0}^{*}=U_{0}\left(e^{\mu_{1} a_{0}}-1\right)+(B-1) \int_{0}^{w_{0}} u_{0}(w)\left(e^{\mu_{1} R(w)}-1\right) d w \text { and } \sigma=B e^{-\mu_{1} a_{11}} .
\end{gathered}
$$

Hence as $n \rightarrow \infty$

$$
u\left(w, n a_{0}\right) \rightarrow \begin{cases}\frac{\mu_{1}\left(B-e^{\mu_{1} a_{0}}\right)}{\mu_{2} U_{0}^{*}} u_{0}(w) & \text { if } B e^{-\mu_{1} a_{0}}>1, \\ 0 & \text { if } B e^{-\mu_{1} a_{14}}<1 .\end{cases}
$$


Thus the population will converge to a periodic function if $\mu_{1} a_{0}<\ln B$. Otherwise, the population will eventually die out. Note that this condition depends on the growth rate $r(w)$ only through the cell cycle duration time $a_{0}$.

Figure 7 shows the solutions for a "top hat" initial condition and two different growth functions. In Figure 7a the cells undergo exponential growth, $r(w)=1+\alpha w$, where $\alpha=0.1$. Again, the initial condition becomes stretched as it propagates along the characteristic curve. In Figure $7 \mathrm{~b}$ the cells undergo von Bartalanffy-type growth, $r(w)=1+\alpha w$, where $\alpha=-0.1$. Here the shape of the characteristic compresses the "top hat" as it travels. In both cases $\mu_{1} a_{0}<\ln B$ and, as stated previously, the solutions do not converge to the steady state but instead to the periodic solution (2.18), with period $a_{0}$.

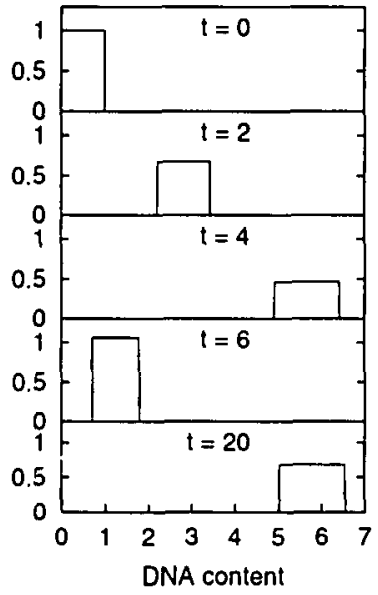

(a)

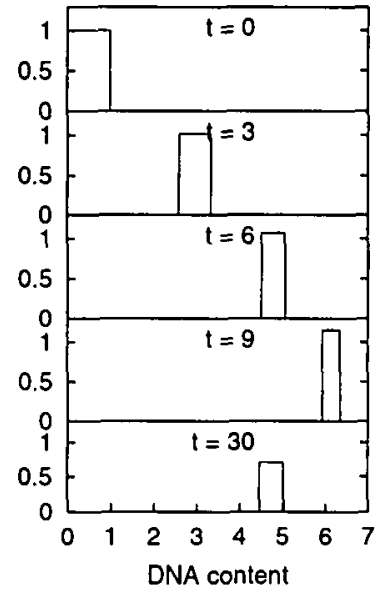

(b)

FIGURE 7. Cell population density against DNA content at successive values of time with a delta birth function, $b(w)=B \delta\left(w-w_{0}\right)=2 \delta(w-7)$. The mortality rates are $\mu_{1}=\mu_{2}=0.05$. The growth rate is $r(w)=1+\alpha w$ : (a) $\alpha=0.1$ (exponential growth); (b) $\alpha=-0.1$ (von Bartalanffy-type growth). In both cases, the solution converges to a periodic function of period $a_{0} \approx 5.3$ in (a) and $a_{0} \approx 12.0$ in (b).

\section{Discussion}

A specific case of the von Foerster equation has been considered in which the mortality rate is a sum of a function of the total population (which models the constant background rate plus death due to overcrowding or competition for resources) and a function of weight (which may represent, for example, the removal of a parent after 
reproduction, as in cell division). The birth rate was taken to be an arbitrary function of age, but independent of the total population size.

A general solution method was presented which reduced the nonlinear integropartial differential equation to a first-order ordinary differential equation. Specific choices of mortality dependence on total population were then presented in which this equation could be solved explicitly; these included the commonly considered case of linear dependence.

Specific solutions were then presented for particular choices of birth function. These included the case of constant birth rate (for both constant and non-constant growth rates), in which it was shown that the population will survive in the long term if the birth rate plus the slope of the linear growth rate is greater than the death rate. We then considered the case in which reproduction occurs at a given age or size, so that the birth rate is a $\delta$-function. We first examined the case in which the parent survives and continues to be a drain on resources, and then looked at the case in which the parent is removed after reproduction, which is appropriate when modelling populations of cells. We found that, in general, the population of cells will evolve towards a periodic solution if the death rate multiplied by the cell cycle duration is less than $\ln B$, where $B$ is the number of daughter cells at division (in general $B=2$ ). The period of the solution is the natural duration of the cell cycle. If the product exceeds $\ln B$, then the population will eventually die out.

In all cases, death due to overcrowding does not impact on the long-term survival versus extinction of the population, but, in the event the population survives, does affect its eventual size.

\section{References}

[1] B. Alberts, D. Bray, J. Lewis, M. Raff, K. Roberts and J. D. Watson, The molecular biology of the cell, 3rd ed. (Garland, New York, 1994).

[2] B. Basse, B. Baguley, E. Marshall, G. Wake and D. Wall, "Modelling the flow cytometric data obtained from unperturbed human tumour cell lines: Parameter fitting and comparison.", Bull. Math. Biol. 67 (2005) 815-830.

[3] B. Basse, B. C. Baguley, E. S. Marshall, W. R. Joseph, B. van Brunt, G. Wake and D. J. N. Wall, "A mathematical model for analysis of the cell cycle in cell lines derived from human tumours", $J$. Math. Biol. 47 (2003) 295-312.

[4] B. Basse and P. Ubezio, "A generalised age and phase structured model of human tumour cell populations both unperturbed and exposed to a range of cancer therapies", Bull. Math. Biol. 69 (2007) 1673-1690.

[5] B. Basse, G. C. Wake, D. J. N. Wall and B. van Brunt, "On a cell-growth model for plankton", Math. Med. Biol. 21 (2004) 49-61.

[6] L. W. Botsford, B. D. Smith and J. F. Quinn, "Bimodality in size distributions: The red sea urchin Strongylocentrotus franciscanus as an example", Ecol. Appl. 4 (1994) 42-50. 
[7] P. Chen, D. Brenner and R. Sachs, "Ionizing radiation damage to cells: effects of cell cycle redistribution", Math. Biosci. 126 (1994) 147-170.

[8] D. A. Coombes, R. P. Duncan, R. B. Allen and J. Truscott, "Disturbances prevent stem size density distributions in natural forests from following scaling relationships", Ecol. Lett. 6 (2003) 980-989.

[9] J. M. Cushing, "Existence and stability of equilibria in age-structured population dynamics", Math. Biol. 20 (1984) 259-276.

[10] J. Folkman, "Tumour angiogenesis: therapeutic implications", New Engl. J. Med. 285 (1971) 1182-6.

[11] M. Gurtin and R. MacCamy, "Some simple models for nonlinear age-dependent population dynamics", Math. Biosci. 43 (1978) 199-211.

[12] R. Hilborn and C. J. Walters, Quantitative Fisheries Stock Assessment (Chapman and Hall, London, 1992).

[13] F. Hoppensteadt, Mathematical theories of populations: demographics, genetics and epidemics (SIAM, Philadelphia, 1975).

[14] A. McKendrick, "Applications of mathematics to medical problems", Proc. Edinburgh. Math. Soc. 44 (1926) 98-130.

[15] J. D. Murray, Mathematical biology (Springer-Verlag, Berlin, 1989).

[16] Norhayati and G. C. Wake, "The solution and stability of a nonlinear age-structured population model", ANZIAM J. 45 (2003) 153-165.

[17] F. Sharpe and A. J. Lotka, "A problem in age distribution", Phil. Mag. 21 (1911) 435-438.

[18] T. Takada and H. Caswell, "Optimal size at maturity in size-structured populations", J. Theor. Biol. 187 (1997) 81-93.

[19] H. von Foerster, "Some remarks on changing populations", in The kinetics of cell proliferation (ed. F. Stohlman), (Grune and Stratton, New York, 1959) 382-407.

[20] G. Webb, Theory of nonlinear age-dependent population dynamics (Dekker, New York, 1985). 